\title{
Developing a system to monitor family planning and HIV service integration: results from a pilot test of indicators
}

\author{
Susan E Adamchak, ${ }^{1}$ Francis O Okello, ${ }^{2}$ Inoussa Kaboré ${ }^{3}$
}

\begin{abstract}
${ }^{1}$ RH/HIV Evaluation Consultant, Manhattan, KS, USA

${ }^{2}$ Deputy Research Director, Abt Associates, Inc., Washington DC, USA

${ }^{3}$ Regional Chief, Monitoring and Evaluation, UNICEF West and Central Africa Regional Office, Dakar, Senegal
\end{abstract}

\section{Correspondence to} Dr Susan E Adamchak, RH/HIV Evaluation Consultant, 1000 Mill Valley Circle, Manhattan, KS 66554, USA; sadamchak@kansas.net

Received 25 July 2014 Revised 7 May 2015 Accepted 21 August 2015 Published Online First 30 September 2015
CrossMark

To cite: Adamchak SE, Okello FO, Kaboré I. J Fam Plann Reprod Health Care 2016;42:24-29.

\begin{abstract}
Objectives To determine the feasibility of collecting data to calculate six indicators of family planning (FP) and HIV integration using existing health information systems (HIS); obtain information to refine indicators; and identify changes needed in existing HIS to calculate indicators.

Design Data abstraction from HIS in a purposive sample of 39 health facilities in Ethiopia, Rwanda, Tanzania and Uganda to analyse data availability and quality undertaken between November 2010 and March 2011.
\end{abstract}

Methods Teams reviewed patient record cards, registers, monthly and quarterly reports for the most recent complete month. Teams recorded all possible sources for each data element, indicated whether data are collated monthly, and whether disaggregation by age, sex and originating service was possible.

Results With the exception of Uganda, all countries were able to report the proportion of service delivery points offering integrated services. Ethiopia was able to calculate the indicator for fixed sites but not for homebased care services. In most cases we were able to calculate the proportion of HIV clients receiving FP services or referral, and the proportion of FP clients receiving an HIV test or referral. It is feasible to collect data for these indicators under current circumstances in some countries.

Conclusions Several actions are proposed for national health systems, including adopting a system of unique client identifiers. Age group reporting bands should be aligned across services. More accurate counts of daily and active client loads are needed to provide programme managers with information to inform programme monitoring.

\section{Key message points}

- In most cases it is feasible to collect data on the proportion of HIV clients receiving family planning (FP) services/ referral, and FP clients receiving an HIV test/referral.

- Services need to develop a better understanding of their daily and active client loads to assist programme managers in allocating resources effectively and efficiently.

- Adopting a system of unique client identifiers is proposed for national health systems.

\section{INTRODUCTION}

National HIV/AIDS programmes are increasingly considering diverse ways in which complementary reproductive health services can be delivered efficiently and effectively. International donor agencies explicitly encourage the integration of family planning (FP) services and HIV programmes as one strategy to address the high number of unintended pregnancies occurring among women living with $\mathrm{HIV}$, and to reduce rates of vertical transmission of HIV. ${ }^{1-3}$ There is a growing body of literature that describes the effect of integrated FP/HIV services introduced through pilot studies or as components of clinical trials, but routine measurement of regular programme implementation is essential. $^{4-7}$ However, efforts to record routine services have been hampered by the lack of standardised indicators that 
measure programme reach and outcomes. Limited data hinder improving service delivery as benchmarks against which to monitor performance are lacking.

The past decade has seen an unprecedented level of international collaboration in the identification and the application of common indicators to track progress in the achievement of health and development goals. If the momentum to promote integrated services including those linking FP and HIV as a standard of practice continues, it is important that appropriate indicators are identified through a systematic and rigorous process to foster common reporting within and among countries. Facilitating this process, a compendium of indicators was published in 2014 to assess sexual and reproductive health and HIV linkages at the policy, service delivery and system levels using data derived from national statistics and regular facility assessments. ${ }^{8}$

FHI 360, a multi-sector development organisation, supports more than 20 Ministries of Health $(\mathrm{MOH})$ in the implementation, monitoring and evaluation of national HIV prevention, and care and treatment programmes, including some with integrated FP/HIV services. This multi-country presence with locally based monitoring and evaluation (M\&E) teams offers an efficient platform in which to test the feasibility of collecting the data needed to calculate indicators.

The goal of this pilot test was to determine which indicators relevant to integrated FP/HIV services could be populated with data already being collected using $\mathrm{MOH}$ registers and current data recording procedures. It had the following objectives:

- To determine the feasibility of collecting data needed to calculate integration indicators

- To obtain information needed to refine the indicators

- To identify changes to existing health information systems (HIS) needed to calculate indicators.

In addition, we assessed data availability; completeness of data; and whether it was possible to disaggregate for key demographic characteristics.
METHODS

Indicator development process

In 2008, an FHI 360 working group consisting of M\&E experts and researchers convened to develop indicators to capture the scope of FP/HIV integration efforts, specified according to international guidelines, ${ }^{9}$ and to identify data sources and appropriate methods of data collection. The group considered the quality and complexity of existing data collection systems, provider motivation to maintain data without increasing daily burdens of work, and not increasing the reporting burden for M\&E staff. These factors were balanced against the need to produce indicators likely to influence national and global policy. Fourteen indicators were assessed using three criteria: (1) whether data were collected for the proposed indicators; (2) the possibility of modifying existing data collection tools to include indicators not currently captured; and (3) whether national HIS include the indicators in their data collection tools. Subsequently, six indicators were selected for assessment (Table 1).

The working group specified each indicator's rationale, definition, numerator, denominator, level of disaggregation, reporting frequency, and calculation. Full specifications of indicators are available upon request from the authors.

\section{Pilot test}

Eligibility criteria and country selection

FHI 360-supported programmes that include integrated services and met the following three conditions were eligible to participate in the pilot test:

1. $\mathrm{MOH}$ supports integrated $\mathrm{FP} / \mathrm{HIV}$ services, including large pilot studies

2. It is feasible to collect data for at least four of the six indicators

3. Integrated services are provided in a minimum of 10 service delivery sites.

Four countries were selected: Ethiopia, Rwanda, Tanzania and Uganda. Where more than 10 sites were

Table 1 Integration indicators and definitions

\begin{tabular}{ll}
\hline Indicator & Definition \\
\hline $\begin{array}{l}\text { Proportion of HIV-related service delivery points with FP/HIV } \\
\text { integrated services }\end{array}$ & $\begin{array}{l}\text { Proportion of HIV-related SDPs providing FP screening, counselling, commodities and/or } \\
\text { referrals through any relevant service such as care and treatment, HIV testing and } \\
\text { counselling, etc. } \\
\text { Proportion of female and male HIV-related service clients of reproductive age screened for }\end{array}$ \\
$\begin{array}{l}\text { Proportion of HIV-related service clients screened for } \\
\text { FP need }\end{array}$ & $\begin{array}{l}\text { Proportion of female clients of reproductive age attending HIV-related SDP with unmet } \\
\text { Proportion of female clients of reproductive age attending } \\
\text { HIV-related SDPs with unmet need for FP }\end{array}$ \\
$\begin{array}{l}\text { Proportion of HIV-related SDP clients who received FP } \\
\text { method or referral }\end{array}$ & $\begin{array}{l}\text { Proportion of HIV-related SDP clients of reproductive age with unmet need who received FP } \\
\text { method (condoms, pills, injectable, implant) or referral after FP counselling }\end{array}$ \\
Proportion of C\&Tx clients reporting unintended pregnancy & $\begin{array}{l}\text { Proportion of continuing female C\&Tx clients reporting unintended (unplanned or mistimed) } \\
\text { pregnancy }\end{array}$ \\
Proportion of FP clients who received HIV testing at the FP & $\begin{array}{l}\text { Proportion of female and male FP clients who received HIV test at the FP SDP or were } \\
\text { referred for HIV testing elsewhere }\end{array}$ \\
\hline SDP or were referred for HIV testing &
\end{tabular}

C\&Tx, care and treatment; FP, family planning; SDP, service delivery point. 
Table 2 Participating countries and number of sites

\begin{tabular}{lc}
\hline Country & Sites $(\boldsymbol{n})$ \\
\hline Ethiopia & 7 \\
Rwanda & 11 \\
Tanzania & 8 \\
Uganda & 13 \\
Total & 39 \\
\hline
\end{tabular}

available, sites were purposively selected based on whether they had offered services for at least 6 months. Data collection was completed at 39 sites (Table 2). The number of sites offering services was a selection criteria for country participation; due to site refusals to participate (Ethiopia) and staff constraints (Tanzania), we did not abstract data from 10 sites in these countries.

We designed a standardised data collection form in Microsoft Excel listing 12 elements needed to calculate four of the indicators, noting the data value, its source, date of most recent data, whether data are aggregated monthly, whether data can be disaggregated by age, sex, and originating services, and observations about data quality, such as whether entries were complete, up to date, and recorded correctly in line with the HIS guidelines. [NB. The first indicator, proportion of service delivery points offering integrated services, is derived from programme records. The fifth indicator, proportion of unintended pregnancies among HIV-infected women in continuing care, was included on the data abstraction form, but with the recognition that it is better measured using survey methods.] Fieldwork took place between November 2010 and March 2011. Teams reviewed all $\mathrm{MOH}$ registers and ad hoc adaptations related to HIV services, including HIV counselling and testing (HCT), provider-initiated counselling and testing, care and treatment (C\&Tx), and prevention of motherto-child transmission (PMTCT). Teams also examined registers in FP, antenatal care, labour and delivery, and postnatal care

Teams also reviewed patient record cards, monthly and quarterly reports, and in Tanzania, reports from the HIV electronic record system. Data were collected for the most recent complete month recorded, from all possible data sources for each data element.

\section{RESULTS}

\section{Data availability for indicator calculation}

Indicator 1: Proportion of integrated service delivery points

We anticipated few problems in obtaining data to calculate the indicator as it is derived from programme monitoring sources. Rwanda and Tanzania easily calculated the indicator using programme records. Uganda supports integrated services through community-based distribution, and hence did not have appropriate numerators and denominators. Ethiopia supports a mix of clinic-based PMTCT sites, and at the time of data collection, home-based care with referrals for FP services. The latter is not well captured with this indicator.

Indicator 2: Proportion of HIV-related service clients screened for FP need

No sites recorded screening for FP need in a service register. In three sites visited in Uganda, screening was recorded on the client's HIV Care/antiretroviral (ART) continuation card, and another used a form developed as part of a pilot project with an international partner. At the time of the study, Tanzania was in the process of revising its National HIV Care and Treatment Patient Record Card (CTC-2), to which a column has been added to record FP screening at each clinical encounter.

Indicator 3: Percentage of female clients of reproductive age (15-49 years) attending HIV-related service delivery points with unmet need for FP

Screening for FP need does not take place systematically and is not recorded. Therefore, the numerator for this indicator could not be defined at any site. No country maintained records on women who were determined to have unmet need for FP.

Indicator 4: Proportion of HIV-related service clients who received a FP method or referral after FP counselling

Several different models of service delivery are used, including direct provision of FP in HIV services and referral to co-located or networked FP services. In some cases, HIV-positive clients who receive an FP method are recorded in the FP service register, rather than an HIV C\&Tx register, with neither attribution that they came from HIV services nor a notation of their HIV status. In the few instances in which FP use is recorded, for example in HIV client charts, it is impossible to determine if women are receiving methods for the first time from a C\&Tx provider, or if they switched from a previously source. Virtually no FP service recorded male clients, the exception being a small number of vasectomy cases in Rwanda.

Documentation of referrals is particularly weak, as verbal referrals typically are not recorded. Tanzanian sites were an exception with good referral tracking, and completed referral forms were collated and crossmatched between the FP and HIV services monthly.

Indicator 5: Percentage of continuing C\&Tx clients reporting unintended pregnancy

We were aware that the data to calculate the indicator are more appropriately collected in a special survey of pregnant or recently delivered HIV-positive women. Nevertheless, we wished to see if documentation of unintended pregnancies was taking place informally. As anticipated, none of the sites recorded the intendedness status of pregnancy among HIV-positive women. 
Indicator 6: Proportion of FP clients who received HIV test at the FP service delivery point or were referred for HIV test

Information on HIV testing of FP clients was available in $56 \%$ of sites. Five sites did not offer HIV testing for FP clients, and the remaining sites (34 sites) did not record testing if done. Often a column for testing status or referral was included in the FP service register. In other cases, the FP service shared an HIV testing register with other services. This posed two challenges to allocating those tested to the correct service. First, each service was allotted a fixed number of pages in the book. Once those pages were filled, the same service would jump forward in the register to a new location, raising the possibility that earlier recorded cases might be overlooked. In the other situation, all clients tested were recorded sequentially, regardless of service of origin. No identifying patient number was included, so individuals could not be matched to the originating service.

\section{Summary}

Table 3 summarises our experience in obtaining the data necessary to calculate the indicators. With the exception of Uganda, all countries were able to report the proportion of service delivery points offering integrated services. Ethiopia was able to calculate the indicator for fixed sites but not for its home-based care services. In most cases, we were able to identify data sources necessary to calculate the proportion of HIV clients receiving FP services, though less so for those receiving referrals. In just over half the sites we were able to identify data sources that permit calculation of the proportion of FP clients receiving an HIV test or referral. It was challenging to identify relevant data sources in Rwanda due to the proliferation of service registers, which include duplicate, unreconciled data.

\section{Data limitations}

Defining denominators

Several sites were unable to determine their monthly client loads from their existing records. One factor contributing to this is the use of longitudinal registers, in which clients are tracked over time through a single entry rather than recorded on a daily basis. This is appropriate from a clinical standpoint, but makes compiling data on the volume of users of different services complex. There were also instances in which people were recorded in registers only if they received ARVs, and not if they came for routine checkups. In these cases client folders had to be physically counted in order to determine the number of people attending HIV services monthly. All of the countries recorded cumulative clients enrolled in C\&Tx services, regardless of whether the clients had died, transferred to a different health facility, or had been lost to follow-up. They were frequently unable to disaggregate these cumulative totals to report their active client load.

\section{Multiple sites recorded in register}

There were several cases in which data from other locations were entered into registers, skewing the apparent size of case loads. We also found instances in which data collected from one-time events, such as mobile outreach, were entered into the clinic registers, resulting in atypical rates of service use.

\section{Age groups}

Given the international standard of classifying women aged 15-49 years as being of childbearing age, it is important to be able to disaggregate this group from clinic data in order to calculate measures related to FP and HIV. However, we identified a number of irregularities in recording age that would make this an unnecessarily complex and unwieldy process. Even within the same register in the same clinic, age was recorded variously as age in years, date of birth and year of birth. There were several instances in which different services used different age groups for data aggregation, making it difficult to compare client loads across services. In Tanzania we observed different versions of HIS software in use where different age bands were used for aggregate data. Finally, there

Table 3 Feasibility of calculating indicators by country

\begin{tabular}{|c|c|c|c|c|}
\hline Indicator & Ethiopia & Rwanda & Tanzania & Uganda \\
\hline Integrated service delivery points & $x$ & $x$ & $x$ & NA \\
\hline HIV clients screened for FP need & - & - & - & - \\
\hline Female HIV clients with unmet need for FP & - & - & - & - \\
\hline HIV clients who received FP method or referral & $x$ & * & $x$ & $x$ \\
\hline Unintended pregnancy & - & - & - & - \\
\hline FP clients tested for HIV or referred for testing & $x$ & * & $x$ & $x$ \\
\hline
\end{tabular}

NA: The proportion of integrated service delivery points was not calculated in Uganda due to the programme focus on community health workers.

$X$ : Able to calculate indicators based on existing data.

-: Unable to calculate indicators due to lack of data.

*: Facilities record data needed to calculate the indicators, but in a format that requires substantial investment in time to locate, collect and aggregate the data.

$\mathrm{FP}$, family planning. 
were some instances in which clients were recorded without specifying their age, making it impossible to determine the number of adults of reproductive age.

\section{Unique client identification numbers}

The ability to track clients across the various services in a health facility is essential to documenting the delivery of integrated services. With the exception of Ethiopia, we found few instances in which facilities used a single, unique ID number for clients, presenting a challenge to track completed referrals across services, or to identify clients noted in different registers as the same individual.

\section{DISCUSSION}

The findings of this pilot test indicate that it is feasible to collect data for some, but not all, of the selected indicators of $\mathrm{FP} / \mathrm{HIV}$ integration. Based on these findings, we recommend that three indicators be routinely collected:

- The proportion of service delivery points that offer integrated services (programme data)

- The proportion of HIV-positive women receiving a FP method (ART or FP register)

- The proportion of women accessing FP services who are tested for HIV (FP register).

Generally data are available to calculate these indicators, though there were some cases in which it was difficult to determine correct denominators. Additional thought must be given to adapting the indicator specification of service delivery points to accommodate community-based and mobile services that combine FP provision and HIV support.

Data on referrals are typically poor or non-existent; until such reporting improves, we recommend that programmes only report on services provided and documented for the latter two indicators.

The poor documentation of completed referrals will likely bias the accuracy of these two indicators. Reported values may undercount actual services to the extent that referrals are not recorded or completed. Should referrals be retained in the indicator definition, given that in many cases referrals simply mean sending a woman down the hall to the FP room and thus there is a high likelihood of completion? Or, should we focus only on women receiving an FP method, possibly underestimating women who do have their FP needs met through their contact with HIV services?

We recommend limiting the two latter indicators to focus on women only at the present time. The majority of non-condom FP methods dispensed target women, who often receive them in mother and child health services, infrequently accessed by men.

We also propose including an additional indicator at a future date, namely "the proportion of women accessing HIV services who are screened for unmet FP need", once tools for systematic screening are more widely available and more commonly used. Tools should ascertain that a woman is of reproductive age, ask whether she is sexually active, currently using an FP method, and if not, does she wish to become pregnant.

\section{CONCLUSIONS}

These results demonstrate that it is possible to collect useful data documenting delivery of integrated $\mathrm{FP} /$ HIV services at the facility level, for the most part using existing systems. Nevertheless, the practice of recording data in multiple registers adds a layer of complexity to data extraction, and causes confusion as to which source should be considered primary. When longitudinal registers are used, without a simultaneous daily activity register, information on daily service loads is difficult to obtain.

Several actions are proposed for national health systems. These include moving to a system of a unique identifier for clients, so that cross-service referrals can be tracked and completed referrals more easily documented. Also, efforts should be made to align age group reporting bands across services, recognising that different services may require different age disaggregation to support their programmes. Finally, services need to develop more accurate counts of their daily and monthly active client loads. Daily counts of the numbers of clients seen at the health facility, disaggregated by age and sex, will provide programme managers with useful information that will inform programme monitoring and planning.

Acknowledgements Support for this manuscript was provided by FHI 360 with funds from the USA Agency for International Development (USAID), Cooperative Agreement Number GHO-A-00-09-00016-00, although the views expressed in this publication do not necessarily reflect those of FHI 360 or USAID.

Funding Support for this manuscript was provided by FHI 360 with funds from the USA Agency for International Development (USAID), Cooperative Agreement Number GHO-A-00-09-00016-00.

Competing interests None declared.

Ethics approval The FHI 360 Office of International Research Ethics reviewed this project, and determined that it did not constitute 'human subject research' as defined under DHHS regulation 45 CFR 46.102 (f).

Provenance and peer review Not commissioned; externally peer reviewed.

\section{REFERENCES}

1 State Department, Office of the United States Global AIDS Coordinator. The U.S. President's Emergency Plan for AIDS Relief: Five-Year Strategy. December 2009. http://www.pepfar.gov/ documents/organization/133035.pdf [accessed 14 June 2014].

2 U.S. Government. Implementation of the Global Health Initiative: Consultation Document. February 2010. http://www. pepfar.gov/documents/organization/136504.pdf [accessed 14 June 2014].

3 World Health Organization (WHO). Strategic Considerations for Strengthening the Linkages between Family Planning and 
HIV/AIDS Policies, Programs and Service. 2009. Geneva, Switzerland.

4 Adamchak S, Ntwali Nsengiumva P, Umurerwa L, et al. Measuring and reporting on family planning and HIV service integration. Presentation made at the Sixth Annual Conference for Exchange and Research on HIV/AIDS, 9-10 June 2011, Kigali, Rwanda.

5 Spaulding AB, Brickley DB, Kennedy C, et al. Linking family planning with HIV/AIDS interventions: a systematic review of the evidence. AIDS 2009;23(Suppl. 1):S79-S88.

6 Bradley H, Gillespie D, Kidanu A, et al. Providing family planning in Ethiopian voluntary HIV counseling and testing facilities: client, counselor and facility-level considerations. AIDS 2009;23(Suppl. 1):S105-S114.
7 Chabikuli NO, Awi DD, Chukwujekwu O, et al. The use of routine monitoring and evaluation systems to assess a referral model of family planning and HIV service integration in Nigeria. AIDS 2009;23(Suppl. 1):S97-S103.

8 United Nations Population Fund (UNFPA). SRH and HIV Linkages Compendium: Indicators and Related Assessment Tools. 2014. http://www.unfpa.org/publications/srh-and-hivlinkages-compendium-indicators-and-related-assessment-tools [accessed 14 June 2014].

9 UNAIDS. Indicator Standards: Operational Guidelines for Selecting Indicators for the HIV Response. January 2010. http://www.unaids.org/sites/default/files/sub_landing/files/ 4_3_MERG_Indicator_Standards.pdf [accessed 14 June 2014]. 